\title{
EXOTICS SEARCHES WITH ATLAS
}

\author{
Andrii Tykhonov ${ }^{a}$ \\ Jozef Stefan Institute, 1000 Ljubljana, Slovenia \\ (on behalf of the ATLAS collaboration)
}

Abstract. An overview is presented for the non-SUSY searches for New Physics with the ATLAS detector. The results presented use data collected at centerof-mass energies of $\sqrt{s}=7 \mathrm{TeV}$ and $\sqrt{s}=8 \mathrm{TeV}$, for data sets corresponding to a variety of integrated luminosities. Searches using leptons, photons, missing transverse energy, and jets are performed, as well as searches requiring custom jet and track reconstruction, and searches for the so-called lepton jets. No deviations from Standard Model expectations are observed, hence constraints are placed on the phase space of available theoretical models.

\section{$6 \quad 1$ Introduction}

7 The Standard Model (SM) remains a remarkably successful theory of Physics, s withstanding tests of unprecedented precision. Recent discovery of a Higgs 9 boson, reported by the ATLAS and CMS collaborations [1] has revealed the 10 last missing component of the SM, the scalar field which is predicted in the ${ }_{11} \mathrm{SM}$ and which is responsible for the fact the fundamental particles in the SM obtain their mass. However, there are numerous indications that at the ener13 gies slightly above electroweak cutoff $(1-10 \mathrm{TeV})$ the SM should be altered by 14 more profound theory, New Physics (NP), for which the SM is as an effective 15 low-energy approximation. Most frequently, it is understood in the context of 16 so-called hierarchy problem, i.e. some as yet unknown physics is required to 17 address the stability of electroweak scale against the Planck scale. Next, the 18 SM by construction does not address gravity. Furthermore, it does not accommodate the Dark Matter (DM) and Dark Energy (DE), the existence of which is suggested by various astrophysical observations. Finally, the SM does not explain non-zero neutrino masses.

A plethora of extensions to the SM, known as Exotic models, have been suggested to address these questions. This document covers a variety of searches performed with the ATLAS detector at the Large Hadron Collider (LHC), interpreted in the context of 'exotic' model predictions, including theories with extra spatial dimensions, Hidden Sector theories, vector-like quark models, and seesaw neutrino mass models. Supersymmetry (SUSY) is not considered, except for cases where SUSY is part of a larger extension to the SM, such as the Hidden Sector scenario. A detailed description of the ATLAS detector can be found elsewhere [2]. It is impossible to cover all ATLAS Exotic analyses in the brief conference proceedings. Therefore, only those ATLAS Exotic searches that the author finds most relevant for the focus of the international Lomonosov conference on elementary particle physics, are considered below.

\footnotetext{
${ }^{a}$ E-mail: andrii.tykhonov@cern.ch
} 

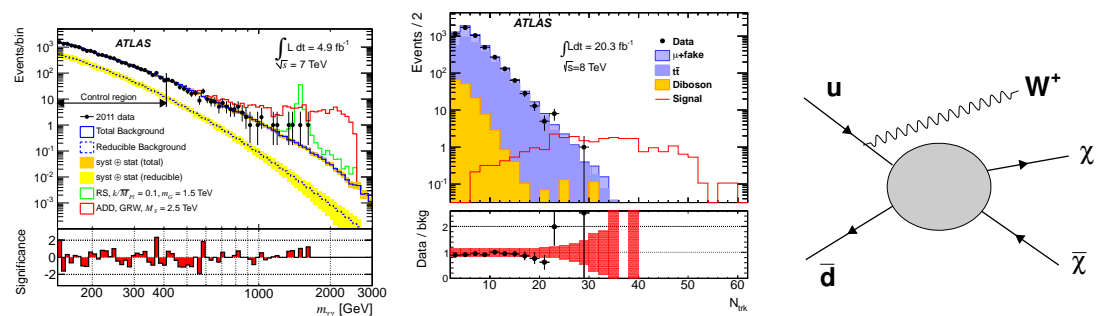

Figure 1: Illustrations of searches for extra dimensions and dark matter: (a) invariant mass of photon-photon pair, where possible signals from different extra-dimension models are shown with red and green histograms and are compared with the stacked histogram, showing the background from various SM processes [3]; (b) multiplicity of high-momentum tracks observed in events after the pre-selection requirement of at least two same-charge muons with $p_{T}>100 \mathrm{GeV}$ per event; red histogram shows the possible signal from microscopic black hole [3]; (c) Feynman diagram illustrating the production of two dark-matter particles that escape detection, in association with $W$-boson from initial-state radiation [4].

\section{$34 \quad 2$ Searches for Extra Dimensions}

Models involving extra dimensions provide a plausible solution of the hierarchy problem, explaining why gravity appears to be much weaker than the other interactions. In these models, gravity is diluted by the presence of extra spatial dimensions, which are compactified at some scale, leading to the weakness of gravity in the regular 4 space-time dimensions. The potential signature of extra dimensions at LHC may come from production of massive excited Kaluza-Klein (KK) gravitons. In some cases, gravitons may decay into pairs of SM particles, as predicted by the Randall-Sundrum theories [7], which would lead to the excess of pair-production of SM leptons, photons, jets (from light quarks), photons or top-quarks, above the predicted SM background, as illustrated in Fig.1a. On the other hand, if couplings of graviton to SM particles are suppressed, as suggested by the ADD models [6], gravitons may evade detection, leading to a signature of large missing transverse momentum and an energetic jet, photon, or $W / Z$-boson from initial state radiation. The latter signature may also come from DM production, when a pair of dark-matter particles escapes the detector, as illustrated in Fig.1c. Finally, since the impact parameter of two colliding protons at LHC can be smaller than the higher-dimensional event horizon, microscopic black holes $(\mathrm{BH})$ may be produced. BH decay through the Hawking radiation, leading to excess of events with high multiplicity of high-momentum particles, as shown in Fig.1b. 

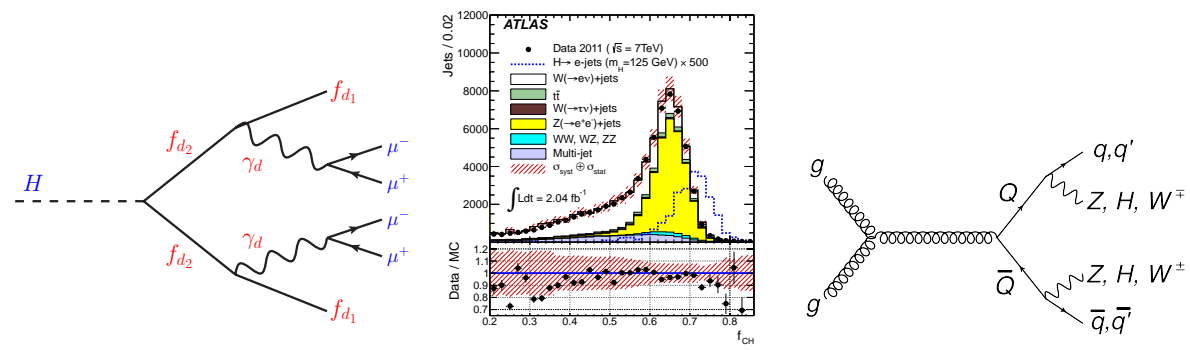

Figure 2: (a) Feynman diagram illustrating production of lepton jets in the decays of a Higgs boson [9]; (b) distribution of jets with respect to charged particles fraction parameter, $f_{\mathrm{CH}}$, in events after $W$-boson selection; contribution from possible lepton-jet signal is shown with the blue histogram (scaled for legibility) [9]; (c) Feynman diagram illustrating production and decay of the pair of vector-like quarks [11].

\section{Dark Matter searches}

${ }_{56}$ Weakly interacting massive particles (WIMPs) is a primary candidate for the

${ }_{57}$ dark matter. Due to their small coupling to SM particles, WIMPs at LHC are

58 assumed to escape detection, leading to a signature of large missing transverse 59 momentum accompanied by energetic jet or photon or gauge boson, similar ${ }_{60}$ to the signatures of extra-dimension scenarios, described above [4]. However, 61 recent astrophysical observations of electron (positron) cosmic-ray spectrum ${ }_{62}$ bring out results which can not be easily explained in conventional WIMP ${ }_{63}$ models [5] and some modifications to these theories are needed, in order to 64 account for anomalous excess of electrons and positrons in cosmic rays. One 65 of the most plausible explanations is presented by the so-called Hidden Sector 66 scenario, which postulates the existence of massive gauge boson of new $U(1)$ 67 group, the dark photon. Dark photons may be produced at LHC, eventually ${ }_{68}$ decaying to pairs of SM electrons, muons or light mesons (mostly charged ones), 69 leading to a signature of so-called lepton-jets. Depending on strength of dark 70 photon coupling to SM photon, lepton jets can be either prompt (originate from 71 interaction point) or produced in displaced vertices (see Fig.2a). Various types 72 of searches for lepton jets are performed at ATLAS [9], exploiting custom-made 73 jet parameters (see Fig.2b).

\section{${ }_{74} 4$ Multilepton searches: origin of neutrino masses}

In the SM neutrinos are massless, which is in contradiction with the evidence from neutrino oscillation experiments, showing that at least two neutrinos of 77 all three generations are massive [8]. In the seesaw mechanism, light neutrino ${ }_{78}$ masses are generated by adding new massive particles to the model. For exam- 
ple, the type III Seesaw mechanism introduces at least two fermionic triplets that generate neutrino masses and the lightest fermionic triplet has two charged heavy fermions, $N^{+}$and $N^{-}$, and a neutral heavy fermion $N^{0}$. Decays of new heavy particles result in events with more than two energetic, prompt and isolated charged leptons. In ATLAS, this type of signatures is probed by looking at events with three or more isolated high- $p_{T}$ electrons (muons), or events with two electrons (muons) with at least one additional $\tau$-lepton [10].

\section{Searches for vector-like quarks}

Vector-like quarks (VLQ) present a viable non-SUSY solution of naturalness problem and are postulated to have both chiralities yielding the same transformation properties under SM $S U(2) \times U(1)$ group. Vector-like top-partner quark, $T$, plays a key role in canceling the quadratic divergences in the Higgs boson mass (induced by top-quark). T-quark, as well as vector-like bottompartner quark, $B$, mixes preferentially with the 3rd generation of SM quarks, whereby signal events feature high multiplicity of jets plus isolated prompt leptons (see Fig.2c). Decays of $T$ and $B$ quarks involve a $W, Z$ or a Higgs boson, hence many complementary analyses are performed, looking at events with high jet multiplicity (including $b$-tagged jets), plus (possibly) isolated high- $p_{T}$ leptons and the requirement of a reasonably high missing transverse momentum [11]. In some signal regions, lepton pairs are required to be consistent with having originated from a $Z$-boson decay [11].

\section{Conslusions}

A wealth of Exotic analyses is underway at the ATLAS experiment. At the time the talk was presented, 53 papers based on 2011 data and 20 conference notes based on 2012 data had been published [12]. This contribution covers only a small fraction of those. So far, no evidence of New Physics was found, and rich (but by far non-exhausting) fraction of phase space of exotic models has been excluded. The new LHC run, which is expected to start in early 2015, poses new challenges in the searches for New Physics, including reconstruction of TeV-scale leptons and heavily boosted objects ( $W$-bosons and top-quarks), investigation of less obvious signatures (lepton jets and displaced vertices), investigation of Higgs sector, and many others.

[1] ATLAS collaboration, Phys. Lett. B, 716:1-29, 2012; CMS collaboration, Phys. Lett. B, 716:30-61, 2012. df

[2] ATLAS collaboration, JINST, 3:S08003, 2008.

[3] ATLAS collaboration, New J. Phys. 15:043007, 2013; Phys. Rev D, 88:072001, 2013. 
[4] ATLAS collaboration, ATLAS-CONF-2013-073; ATLAS-CONF-2012147; ATLAS-CONF-2012-085.

[5] AMS collaboration, Phys. Rev. Lett. 110:14, 2013; Fermi LAT Collaboration, Phys. Rev. Lett. 102:181101, 2009; PAMELA Collaboration, Nature 458:607609, 2009.

[6] N. Arkani-Hamed, S. Dimopoulos, and G. Dvali, Phys. Lett. B, 429:263, 1998; Phys. Rev. D, 59:086004, 1999.

[7] L. Randall and R. Sundrum, Phys. Rev. Lett. 83:3370 1999; Phys. Rev. Lett. 83:4690, 1999.

[8] Super-Kamiokande Collaboration, Phys. Rev. Lett. 81:15621567, 1998; D. Forero, M. Tortola and J. W. F. Valle, Phys. Rev. D86:073012, 2012;

[9] ATLAS collaboration, Phys. Lett. B, 721:32-50, 2013; New J. Phys. 15:043009, 2013; Phys. Lett. B, 719:299-317, 2013.

[10] ATLAS collaboration, ATLAS-CONF-2013-070.

[11] ATLAS collaboration, ATLAS-CONF-2013-018; ATLAS-CONF-2013051; ATLAS-CONF-2013-056; ATLAS-CONF-2013-060.

[12] https://twiki.cern.ch/twiki/bin/view/AtlasPublic/ExoticsPublicResults 